\title{
Quaderni
}

QUADERNI Communication, technologies, pouvoir

69 | Printemps 2009

Universités sous influence du numérique et du management

\section{Travail et apprentissage collaboratifs dans l'enseignement supérieur : opinions, réalités et perspectives}

Éric Bruillard et Georges-Louis Baron

\section{OpenEdition}

\section{Journals}

Édition électronique

URL : http://journals.openedition.org/quaderni/327

DOI : 10.4000/quaderni.327

ISSN : 2105-2956

Éditeur

Les éditions de la Maison des sciences de l'Homme

Édition imprimée

Date de publication : 1 mai 2009

Pagination : 105-113

\section{Référence électronique}

Éric Bruillard et Georges-Louis Baron, «Travail et apprentissage collaboratifs dans l'enseignement supérieur : opinions, réalités et perspectives », Quaderni [En ligne], 69 | Printemps 2009, mis en ligne le 05 avril 2012, consulté le 01 mai 2019. URL : http://journals.openedition.org/quaderni/327 ; DOI : 10.4000/quaderni.327 


\section{Politique}

travail et

apprentissage

collaboratifs dans

l'enseignement

supérieur :

opinions,

réalités et

perspectives

Éric Bruillard

Professeur des universités en informatique UMR Stef, ENS Cachan IUFM de Créteil

Georges-Louis Baron

Professeur en Sciences de l'éducation Université Paris $V$
En une soixantaine d'années, le champ sémantique du mot «collaboration » a passablement évolué. D'abord associé à la collaboration avec l'occupant pendant la Seconde Guerre mondiale (ce n'est pas si ancien), il a pris une valence neutre. Puis il en est venu à acquérir une vertu positive, en particulier dans l'expression « travail collaboratif», surtout s'il est « à distance ».

Pendant cette période de temps, bien des choses ont changé, en effet. Dans l'industrie, les valeurs du travail en commun, de la flexibilité ont été promues, dès les années 80 , avec la diffusion de l'automatistion et le remplacement de chaînes de production par des lignes de fabrications flexibles (par exemple Durand et al, 1987). Le temps est alors venu de la recherche de nouvelles formes de management participatif et de travail en équipe.

Dans le monde universitaire et, plus largement dans les formations supérieures, on parle aussi de travail collaboratif, parce que l'esprit du temps y invite. Dans quelle mesure le met-on en pratique, dans un contexte où les notes sont attribuées aux étudiants sur la base de performances et de productions individuelles et où le travail en commun entre chercheurs fait souvent l'objet d'une division de type hiérarchique?

Nous allons, dans cette contribution, présenter quelques réflexions sur la notion de travail collaboratif en général et la manière dont elle apparaît dans les discours, puis sur son emploi dans les formations supérieures, notamment dans la formation des enseignants, montrant un lien encore problématique avec l'apprentissage collaboratif. 


\section{Travail et apprentissage collaboratifs ?}

Des auteurs comme Dillenbourg (1999) ont établi une distinction entre travail coopératif et collaboratif. Pour aller vite, dans le premier, la division du travail aurait une nature inégale, tandis que la collaboration serait une forme de coopération où les différents collaborateurs ont des rôles similaires dans la conceptualisation des tâches et dans l'intervention commune. La division du travail horizontale y serait instable tandis que la coopération serait fondée sur une division du travail rendue explicite dès le départ. Il y aurait également une forme de symétrie (les membres ont le même statut social et peuvent accomplir les mêmes actions).

Cependant, tout est relatif; le mot collaboration pour un chef de service prend un sens très éloigné, quand il parle de ses « collaborateurs ». Cela nous conduit à nous interroger sur ce qui peut se cacher derrière cette notion de collaboration, qu'elle soit associée au travail ou à l'apprentissage.

Visions et représentations contemporaines $d u$ travail collaboratif

Google est un outil très intéressant dans le sens où les réponses qu'il apporte à des requêtes reflètent les discours qui dominent les débats. Nous avons choisi de faire une comparaison entre les expressions travail collaboratif et apprentissage collaboratif.

Une requête, effectuée le 5 septembre 2008 avec l'expression « apprentissage collaboratif », conduisait alors à 451000 pages (68 600 pour l'expression exacte), alors qu'elle en fournissait 2480000 pour «travail collaboratif» (et 2410000 pour l'expression exacte). En outre, il n'y avait pas de liens commerciaux pour la première requête, alors que la seconde en fournissait 27 (sur 4 pages de résultats). Dans les recherches apparentées, Google oriente essentiellement vers les outils ${ }^{1}$. Ce fait est confirmé par de nombreux écrits du web dans lesquels on voit, par exemple, le wiki apparaître comme le «summum du collaboratif», selon un dossier du Journal du Net ${ }^{2}$.

Les mêmes requêtes effectuées avec le moteur Exalead donnent des résultats similaires ${ }^{3}$, mais avec un facteur de réduction important pour l'expression exacte : 797211 résultats pour « travail collaboratif » (68 932 pour l'expression exacte). Les termes associés proposés (outils de travail collaboratif / espace de travail / plate-forme / outil de travail / solution de travail collaboratif) confirment la confusion avec les outils ou les espaces. «Apprentissage collaboratif » quant à lui recueille 95710 résultats (4 019 pour l'expression exacte $)^{4}$.

Notons qu'un nouvel essai avec Google le 15/09/08 fournit davantage de pages (3 040 000) pour l'expression exacte " travail collaboratif » que pour l'association des deux mots (2 460 000). Résultat inattendu qui conduit à $s$ 'interroger sur le fonctionnement de ce moteur : seraient-ce les liens commerciaux qui orientent l'algorithme à privilégier cette expression? Le grand nombre de pages induit-il des réductions particulières?

La prédominance de l'aspect travail sur l'appren- 
tissage est donc nette s'agissant de « collaboration ». L'expression « travail collaboratif» est devenue une expression toute faite, qui se rabat quasiment sur les outils censés le permettre ou le faciliter.

Ce qui frappe, dans l'exploration des pages proposées, c'est la fréquence de discours stéréotypés, parfois messianiques, insistant sur la nécessité de ce type de travail dans la société moderne, décrivant les offres d'outils pour le développer ou le soutenir, exprimant des déceptions face à l'écart entre les attentes commerciales et les usages repérés... On va maintenant en présenter quelques exemples, parmi bien d'autres, choisis pour leur caractère typique.

Par exemple : "[dans la nouvelle économie] les leviers de création de valeurs et d'innovation sont de plus en plus basés sur la mutualisation des connaissances, l'intelligence collective, l'appropriation par le plus grand nombre des technologies et l'efficacité du travail en réseau $»^{5}$. Ce serait la "gestion par projets, la mobilisation de compétences multiples et la distribution géographique des structures qui conduisent à la nécessité d'outils et d'espaces de travail collaboratif en ligne $»^{6}$, avec l'idée d'instaurer au sein des équipes la « communication par principe », par opposition à la « domination par principe $»^{7}$.

Les discours des formateurs et des consultants s'organisent autour de quelques thèmes: apports pour l'entreprise, fonctionnalités et outils du travail collaboratif, recommandations pour le déployer. Et l'on retrouve la rhétorique classique des cycles de nouveautés technologiques : le contraste entre des utilisateurs « ravis de pousser les solutions de travail collaboratif dans leurs retranchements » et des experts constatant un niveau très bas d'utilisation des fonctionnalités offertes... avec un espoir mis dans les nouvelles générations d'utilisateurs : «Les nouvelles fonctionnalités sont là, poussées par les éditeurs. Mais les usages, eux, émergent lentement. Heureusement, les Millenials arrivent », soit avec de nouvelles offres censées changer la donne ( de nouvelles formes de travail collaboratif arrivent à grands pas, bousculant sur leur passage archaïsmes, freins, barrières et autres habitudes propres à des structures organisationnelles encore trop poussiéreuses $\gg)^{8}$.

Ainsi, « travail collaboratif» renvoie à des pratiques supposées désirables utilisant des outils spécialisés et l'on peut relever une grande homologie avec l'idée du Web dit 2.09. Mais, à y bien regarder, l'ambiguïté entre les différentes valences de la collaboration (partage horizontal ou travail prescrit) est très présente. Et le bilan est plus que mitigé, comme le décrit un peu vertement Serge Levan « Si on parle tant du Web 2.0 et du collaboratif, c'est bien parce qu'il n'y a pas de collaboration, au quotidien, dans l'univers de l'entreprise. Le collaboratif c'est comme la paix dans le monde: tout le monde en parle, mais on ne fait pas ce qu'il faut pour qu'elle devienne une réalité quotidienne. $\rangle^{10}$

\section{Travail collaboratif et e-learning}

\section{Une importation du discours entrepreneurial?}

En milieu universitaire, la e-formation, synonyme moderne de formation à distance, fait lar- 
gement appel au travail collaboratif. C'est ainsi, autour de la e-formation, que le site educnet ${ }^{11}$ propose un dossier sur le travail collaboratif, avec des définitions et des références au Journal $d u$ Net sur les outils ou logiciels collaboratifs.

Dans ce contexte, il semble que le développement de travaux de groupe réponde à des problèmes maintenant bien repérés. Il est notoire que la fourniture de «contenus » d'enseignement est insuffisante : la sensation d'isolement, la difficulté à diriger son propre apprentissage, le manque d'autonomie, etc. conduisent à des taux d'abandon importants. La mise en place de différentes formes de tutorat permet d'assurer davantage de suivi des étudiants dans les dispositifs de formation. Le recours organisé aux chats et aux forums, facilite la création de lien social, en réintroduisant les notions de classe et de groupe et l'apprentissage collaboratif peut apparaître comme un moyen économiquement très intéressant de faire vivre des formations à distance (Bruillard, 2004).

Apprendre à travailler avec d'autres apparaît alors comme un objectif désirable. La question épineuse est alors de savoir comment. On tombe ici sur une sorte d'espace quasi vide. Un tour d'horizon des sites des universités ne donne rien de notable.

Le mot collaboration est cité plusieurs fois dans le rapport au ministre français de l'Enseignement supérieur consacré à l' « université numérique » [Isaac 2008], mais dans le contexte de la mise à disposition de ressources via l'encyclopédie Wikipedia. On trouve une référence au travail collaboratif, dans une section consacrée aux nouvelles modalités de formation, indiquant la nécessité «de repenser la pédagogie classique en face-à-face en articulant des temps en présentiel et du travail asynchrone (forum, blog, wiki, travail collaboratif, etc.) » (op.cit., page 13). La confusion déjà signalée avec les outils est une nouvelle fois attestée, l'expression « travail collaboratif » devenant un équivalent des services informatisés cités.

Finalement, ce serait d'ailleurs en harmonie avec les évolutions actuelles de l'université, on constate ce qui ressemble à une forme d'importation du discours entrepreneurial dans la formation, où les outils sont là comme facilitateurs, prétextes, catalyseurs pour le changement.

Il existe néanmoins des prescriptions liées au travail collaboratif dans l'enseignement supérieur dans le cadre des différents certificats informatique et Internet (C2i) qui sont peu à peu mis en place.

\section{Le cas $d u C 2 i$}

Deux niveaux de C2i sont maintenant proposés. Le travail collaboratif est en bonne place dans chacun d'eux.

Le niveau 1 devrait attester de l'acquisition de compétences générales permettant aux étudiants d'utiliser les TIC dans leur formation. La catégorie B7 du C2 $\mathrm{i}^{12}$ est intitulée « Mener des projets en travail collaboratif à distance. Travailler dans un environnement de travail collaboratif $»$. Elle se décline comme suit :

1. utiliser les outils d'un espace de travail colla- 
boratif (environnement numérique de travail) ;

2. élaborer en commun un document de travail (assurer le suivi des corrections, ajouts et suppressions);

3. gérer différentes versions de documents partagés.

La construction d'un document en commun est l'activité mise en exergue. On est bien dans le travail. La finalité est de s'assurer la maîtrise d'instruments et d'environnements pour des activités dans le cadre universitaire.

Il est intéressant de constater que les cinq certificats de niveau 2 réservent également une place au travail collaboratif ${ }^{13}$. Toutefois, la différence avec le premier niveau n'est pas toujours bien claire.

Ainsi en droit, il est notamment demandé de « rédiger des documents et des actes en commun », en enseignement de "rechercher, produire, partager et mutualiser des documents, des informations, des ressources dans un environnement numérique » et de « contribuer à une production ou à un projet collectif au sein d'équipes disciplinaires, interdisciplinaires, transversales ou éducatives », en santé de " mettre en œuvre une collaboration dans le cadre de ses activités professionnelles » et de "choisir et utiliser à bon escient les outils d'échange et de partage de l'information », enfin en ce qui concerne les «métiers de l'environnement et de l'aménagement durables ", il faut pouvoir "gérer le cycle de vie des documents et leurs versions » et « contribuer à un projet collaboratif professionnel en mettant en œuvre les bonnes pratiques ».

D'un point de vue technique, il n'y a là rien d'autre que de mettre en œuvre les compétences attestées au niveau 1 dans un domaine de spécialité. On pourrait penser que d'autres compétences spécifiques «métier » entrent également en jeu, mais elles ne sont pas spécifiées. S'agit-il d'ailleurs vraiment de faire acquérir des compétences ou bien d'inciter à la mise en place d'activités particulières en formation?

Une mention spéciale peut être adressée aux métiers de l'ingénieur: la dénomination niveau 2 semble être mieux justifiée, puisqu'il s'agit de passer d'un statut d'utilisateur à un statut d'animateur ou de gestionnaire dans le domaine qualifié d' « environnement numérique et ingénierie collaborative » :

\section{Pratiquer les outils d'ingénierie collaborative} de la conception à l'exploitation d'un processus ou d'un produit.

2. Animer un espace collaboratif et mettre en cuvre les bonnes pratiques et le contexte d'usage des outils synchrones et asynchrones.

3. Assurer la bonne gestion des documents et le cycle de vie.

4. Maîtriser les contraintes de travail connecté ou déconnecté et maîtriser la configuration et la sécurité de son accès réseau.

On a donc des prescriptions cohérentes s'agissant de développer une première maîtrise puis de déployer des activités jugées comme essentielles à un métier. Bien évidemment, prescription n'est pas utilisation et la mise en œuvre effective est en question.

En effet, compte tenu de l'organisation actuelle de l'université française, de la taille des groupes 
dans la plupart des filières, des types de travaux demandés aux étudiants, des modalités d'évaluation, dans quelle mesure est-il raisonnable de tabler sur un développement de modalités de travail collaboratif?

Une rapide analyse de la production scientifique suggère que les colloques sur les TIC dans l'enseignement supérieur montrent peu de « retours d'expérience» convaincants (on retrouve en revanche la traditionnelle interrogation sur un déficit d'usage des outils et espaces proposés).

L'injonction institutionnelle, quant à elle, porte davantage sur l'utilisation des ressources que sur leur construction (éventuellement collaborative). Il n'est donc pas étonnant de trouver des offres marchandes, où la consommation remplace la coopération $^{14}$. Ainsi dans le C2i santé, bien que s'agissant de « travail collaboratif en santé », la compétence 2.5 est «Utiliser des ressources en ligne ou des dispositifs de formation ouverte et à distance $»$. Le travail dit collaboratif aurait-il pour objet de préparer les étudiants à de nouvelles modalités de formation tout au long de la vie?

\section{Les formations d'enseignants, formations exemplaires?}

Sans surprise, le cahier des charges de la formation des maîtres ${ }^{15}$, dans la section intitulée «Maîtriser les technologies de l'information et de la communication », introduit la capacité « travailler en réseau avec les outils du travail collaboratif ». Là encore, c'est le travail qui est indiqué avec l'expression rituelle. On peut remarquer que la compétence suivante s'intitule «Travailler en équipe et coopérer avec les pa- rents et les partenaires de l'école ». On retrouve la coopération! Cette dernière expression est plus habituelle dans le champ scolaire, notamment dans la lignée de Célestin Freinet.

Le travail en collaboration ne constitue pas une base historique du travail des enseignants et si la coordination de pratiques peut se prescrire, ce n'est pas le cas pour la collaboration entre enseignants (Marcel et al., 2007). Bien que des réussites exceptionnelles puissent être notées dans l'enseignement de second degré, comme le travail mené au sein de l'association Sesamath ${ }^{16}$ (notamment dans la production d'outils en ligne et de manuels scolaires libres), elles n'ont guère d'équivalent dans l'enseignement supérieur.

Au-delà de la collaboration ou de la coopération, qu'en est-il de formes d'apprentissage collectif, dont on peut constater qu'elles sont loin d'être généralisées ? Nous travaillons depuis plusieurs années sur ce sujet, notamment avec des formateurs de différents IUFM et des chercheurs, initiant des symposiums (Symfonic ${ }^{17}$ ) ou des conférences comme Jocair ${ }^{18}$ et nous pouvons en tirer quelques leçons (Baron et Bruillard, 2006).

Partager des ressources, pour les enseignants, n'est pas un problème majeur. C'est même une nécessité de survie, notamment dans les stages sur le terrain. En revanche, au-delà de la mutualisation, la production commune, dans des situations de travail, l'écoute de l'autre, l'intégration de son point de vue sont des objectifs difficiles à atteindre. Cela se «travaille» en formation, dans des formes de communication à distance, en vue d'une élaboration collective. 
Des modèles sont à élaborer. La recherche s'y emploie, une de ses finalités est d'aider à faire évoluer les idées et les formations et à faire exister d'autres discours que ceux empruntés à la vision dominante de l'entreprise.
$R \cdot \dot{E} \cdot F \cdot E \cdot R \cdot E \cdot N \cdot C \cdot E \cdot S$

BARON Georges-Louis, BRUILLARD Éric (dir.) (2006). Technologies de communication et formation d'enseignants : vers de nouvelles modalités de professionnalisation? Lyon: INRP, 249 pages.

BRUILLARD Éric (2004). Apprentissage coopératif à distance: quelques repères sur les questions de recherche. in Enseignement à distance : épistémologie et usages, Hermès-Lavoisier, pp. 115-135.

BRUILLARD Éric (2008). Travail et apprentissage collaboratifs dans des formations universitaires de type hybride. Éléments de réflexion. Éducation - Formation : Tribune libre d'informations et de discussions pédagogiques -e-288, Septembre 2008, http://ute3.umh.ac.be/revues/ Durand Jean-Pierre, Lévy Pierre, Weissberg Jean-Louis (1987). Guide de l'informatisation. Paris : Belin, 346 pages.

DILLENBOURG Pierre. (1999). What do you mean by collaborative learning? Retrouvé Septembre 14, 2008, de http://hal.archives-ouvertes. fr/index.php?halsid=4qtklf9svci16b5keq107ejt2 $5 \&$ view_this_doc $=$ hal-00190240\&version $=1$. ISAAC Henri (2008). Université numérique. Rapport à Madame Valérie Pécresse, ministre de 1'Enseignement Supérieur et de la Recherche. Site Université numérique, 11 janvier 2008, PDF, 54 pages. http://www.universitenumerique.fr/IMG/pdf/Rapport_univ_num.pdf

MARCEL Jean-François, DUPRIEZ Vincent, PERISSET BAGNOUD Danièle, TARDIF Maurice (2007). Coordonner, collaborer, coopérer - De nouvelles pratiques enseignantes, De Boeck, collection Perspectives en Éducation. 
$\mathrm{N} \cdot \mathrm{O} \cdot \mathrm{T} \cdot \mathrm{E} \cdot \mathrm{S}$

1. Liste des expressions proposées: outils travail collaboratif / travail collaboratif open source / travail coopératif / groupware espace de travail collaboratif / outil de travail collaboratif / travail collaboratif en ligne / logiciel de travail collaboratif

2. http://www.journaldunet.com/management/ dossiers/031115cominterne/wiki.shtml

3. Google Scholar (consulté le 15/09/08) donne des résultats équivalents mais plus resserrés avec respectivement 4210 (2 740 pour l'expression exacte) avec travail collaboratif et 2960 (1 040 pour l'expression exacte) résultats pour apprentissage collaboratif.

4. Avec les termes associés: travail collaboratif / plate-forme / formation continue / bases de données / éducation nationale.

5. http://www.grenoble-em.com/default. aspx?rub=897

6. Société Emencia, http://www.emencia.fr/fr/solutions/travailcollaboratif

7. http://www.journaldunet.com/management/ dossiers/031115cominterne/wiki.shtml. Voir aussi Yvan Potin (2007). Travail coopératif : quand la distance permet le rapprochement, http://www.creg.acversailles.fr/article.php3?id_article=206.

8. http://www.journaldunet.com/solutions/0703/070326-enquete-collaboratif/1.shtml

9. Comme l'indique le titre: «Le Web 2.0 dans l'entreprise : la réinvention du travail collaboratif» (http://www.atelier.fr/applications-it/web,20, entrep rise, reinvention, travail,collaboratif-32495-14; actu. html) ou le commentaire : «En forçant (à peine ?) le trait, j'aurais envie de dire que le travail collaboratif, c'est introduire les méthodes et les outils $d u$ Web 2.0 dans notre quotidien professionnel, avec la multiplication des situations de collaboration que cela induit » (http://www.synergies-publiques.fr/article. php?id_article=780)

10. http://travailcollaboratif.typepad.com/

11. http://www.educnet.education.fr/dossier/eformation/modularite5.htm

12. http://www.education.gouv.fr/bo/2005/15/ MENT0500561C.htm

13. http://www2.c2i.education.fr/sections/c2i2e/referentiel/ pour le référentiel du niveau 2, enseignement. On trouve les autres référentiels sur le même site.

14. Ainsi, on trouve sur le site oodoc, au prix de 3,60 €, un «Exposé technique réalisé en 1ère année de cycle d'ingénieur à l'Institut National des Sciences appliquées (INSA) », intitulé Les technologies de travail collaboratif.

15. Arrêté du 19 décembre 2006 portant cahier des charges de la formation des maîtres en institut universitaire de formation des maîtres, MENS0603181A, JO du 28 décembre 2006.

16. http://www.sesamath.net/

17. http://www.dep.u-picardie.fr/sidir/index.htm

18. http://www.jocair.u-picardie.fr/ 
$R \cdot E ́ \cdot S \cdot U \cdot M \cdot E ́$

La dimension collaborative, qu'il s'agisse du travail ou de l'apprentissage, occupe actuellement une place importante dans les discours sur l'éducation. Que nous disent au juste ces discours, quelles idées dominantes véhiculent-ils? Nous avons mené une analyse exploratoire de ceux qui ont été publiés sur le web et des prescriptions relativement à l'apprentissage. Cette analyse, qui confirme l'existence d'un lien fort de la collaboration avec des idées d'innovation, de modernité et de dynamisme, fait apparaître une situation cependant contrastée et problématique, suggérant que les faits sont encore loin des discours et qu'il y a encore bien du chemin à faire pour que des formes collectives de travail et d'apprentissage jouent un rôle important dans l'enseignement supérieur.

The collaborative dimension, for work or for learning, currently occupies an important place in discourses about education. What are really saying those discourses, what dominant ideas do they convey? We conducted an exploratory analysis of these discourses that have been published on the web and of requirements about collaborative learning. This analysis confirms the existence of a strong link with the ideas of innovation, modernity and dynamism. It however reveals a contrasted and even problematic situation, and suggests that there is still a long way to go before the collaborative forms of work and learning may play an important role in higher education. 
